\title{
The genus Bartramia Hedw. (Bartramiaceae, Bryophyta) in Uruguay
}

\section{El género Bartramia Hedw. (Bartramiaceae, Bryophyta) en Uruguay}

\author{
Guillermo Martín Suárez ${ }^{1,3}$, María Soledad Jiménez² \& Jorge Rafael Flores ${ }^{* 1,3}$ \\ ${ }^{1}$ Unidad Ejecutora Lillo, CONICET - Fundación Miguel Lillo, Miguel Lillo 251, San Miguel de Tucumán (4000), Tucumán, \\ Argentina. \\ ${ }^{2}$ Instituto de Botánica del Nordeste (IBONE-CONICET-UNNE), Sgto. Cabral 2131, C.C. 209, Corrientes, Argentina. \\ ${ }^{3}$ Facultad de Ciencias Naturales e I.M.L., Universidad Nacional de Tucumán, Miguel Lillo 205, San Miguel de Tucumán, \\ Tucumán, Argentina. \\ *jrflores@conicet.gov.ar
}

\begin{abstract}
El género Bartramia Hedw. se registra por primera vez para Uruguay. Sólo una especie habita la región de estudio ( $B$. laevisphaera) la cual es caracterizada morfológicamente e ilustrada con fotomicrografías ópticas y electrónicas de barrido. Se presenta un mapa de la distribución de la especie en Latinoamérica.
\end{abstract}

Between 2011 and 2015 botanical surveys were undertaken in Uruguay where significant bryophyte collections were made. Geographically, The República Oriental del Uruguay is situated in southern South America, delimited northward by Brazil, by Argentina to the west and the Atlantic Ocean to the east.

The knowledge of the bryoflora of Uruguay is poor and restricted to a few studies, principally in Montevideo (Matteri 2004). Additionally, new mosses and liverworts have been recently recorded in different areas of the country (Ellis et al. 2011, 2012a, 2012b, Flores \& Suárez 2014, Suárez \& Schiavone 2013, Suárez et al. 2014), which highlights the floristic importance and the singularity of its flora.

Bartramia laevisphaera (Taylor) Müll. Hal. has been recorded in South America from Colombia, Venezuela, Ecuador, Peru, Bolivia, and northwestern Argentina. The first record for the genus Bartramia (with B. laevisphaera) in Tacuarembó, Uruguay, is here presented, along with a distribution map for Latin America, and illustrations.

Bartramia laevisphaera (Taylor) Müll. Hal., Syn. Musc. Frond. 1: 506. 1849.

Glyphocarpa laevisphaera Taylor, London J. Bot. 5: 56. 1846. Glyphocarpus laevisphaerus (Taylor) A. Jaeger, Gen. Sp. Musc. 1: 526. 1875. Anacolia laevisphaera (Taylor) Flowers in Grout, Moss Fl. N. Amer. 2: 155. 1935. TYPE CITATION: Ecuador: on Pichincha; growing on shaded trunks of tree, and on the ground; September 23, 1826. Prof. W. Jameson, (Dr. Greville's herbarium). (Lectotype, selected by Suárez et al. 2012: Ecuador, Pichincha, Jameson 1827, FH!; isolectotype: LIL-Matteri!). Figs. 1-3.
Description. Plants medium-sized, green, to $1.8 \mathrm{~cm}$ high. Stems moderate to strongly tomentose at base, hyalodermis absent, central strand present; axillary hairs two-celled, lower cell quadrate, brown, upper cell globose, hyaline. Leaves appressed when dry, erect-spreading when wet, ovate-lanceolate; apex narrowly acuminate; margins coarsely double-serrate, revolute above; upper cells bistratose, oblong to linear, prorulose at both ends, marginal cells usually shorter, thicker-walled and more highly colored; cells of the marginal teeth much larger; inner basal cells linear, only slightly wider than the upper cells, basal marginal cells becoming much wider and shorter, mostly oblong, extreme marginal ones often quadrate. Sporophytes not observed.

Specimens examined. URUGUAY. Tacuarembó, Gruta de los Cuervos, $31^{\circ} 17^{\prime} 0.7^{\prime \prime} \mathrm{S}$; 56 $06^{\circ} 30.3^{\prime \prime} \mathrm{W}, 220 \mathrm{~m}, 28$-II2012, G. Suárez, M. Dematteis, E. Meza \& A. Vega 1366 (CTES 0586238, LIL 56204). ARGENTINA. Tucumán,

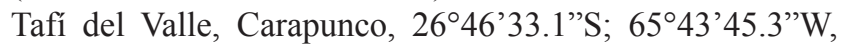
2798 m, 07-VI-2009, G. Suárez 618 (LIL 56205); La Ciénaga, 2647'10”S; 65³9'04”W, 2636 m, 09-IV-2010, G. Suárez 799 (LIL 56208); G. Suárez 809 (LIL 56207). La Rioja, Chilecito, Guachín, en suelo, a orillas del sendero, formando césped, 08-X-2010, N. Canton 921 (LIL 56206). ECUADOR. Cotopaxi, Parque Nacional Cotopaxi, NW slope of volcán Cotopaxi, Laguna Limpia Pongo, dry páramo on slope next to the lake, $0^{\circ} 38^{\prime} \mathrm{S} ; 7^{\circ} 32^{\circ} \mathrm{W}, 3900 \mathrm{~m}$, 25-IX-1988, Frahm \& Gradstein 22 (LIL 22). 
OBSERVATIOns. Found during extensive botanical explorations in Uruguay, the new records of Bartramia laevisphaera are the first report for this genus and species from the country (Fig. 4). The species was found growing in stony grasslands at $200 \mathrm{~m}$ above sea level. In a sterile condition, B. laevisphaera can be confused with B. stricta (Damayanti et al. 2012, Matteri 1983). However, the latter can be distinguished from $B$. laevisphaera by its wide central strand in the stems (occupying 87.5-93.75(-175) $\mu \mathrm{m} ; 22-31 \%$ vs. $50-56.25 \mu \mathrm{m} ; 20-25 \%)$, thinner walls of the stem cortical cells (less than $2 \mu \mathrm{m}$ vs. 2.5-3.7 $\mu \mathrm{m}$ ). Another interesting character observed was the axillary hairs with 1 basal brown and 1 globose distal hyaline cell in B. laevisphaera and 1-2 basal brown and 1 globose to sub-globose hyaline cells in B. stricta.
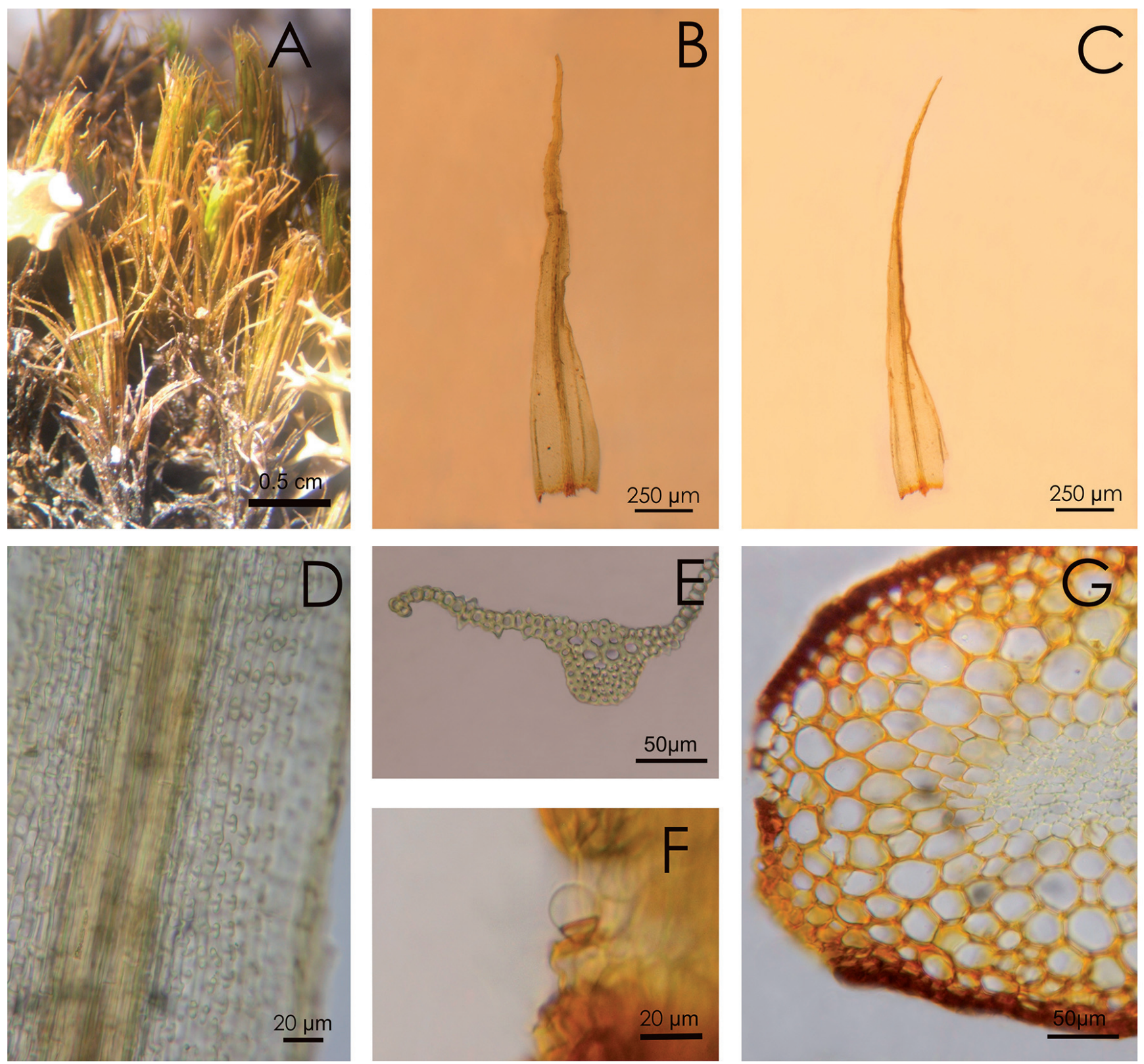

Figure 1. Bartramia laevisphaera A. Habit. B. C. Leaves. D. Median leaf cells. E. Leaf cross section. F. Axillary hair. G. Stem cross section [from G. Suárez et al. 1366 (CTES, LIL)]. / Bartramia laevisphaera A. Habito. B. C. Hojas. D. Células de la lámina media. E. Sección transversal de la hoja. F. Pelos axilares. G. Sección transversal del tallo [de G. Suárez et al. 1366 (CTES, LIL)]. 

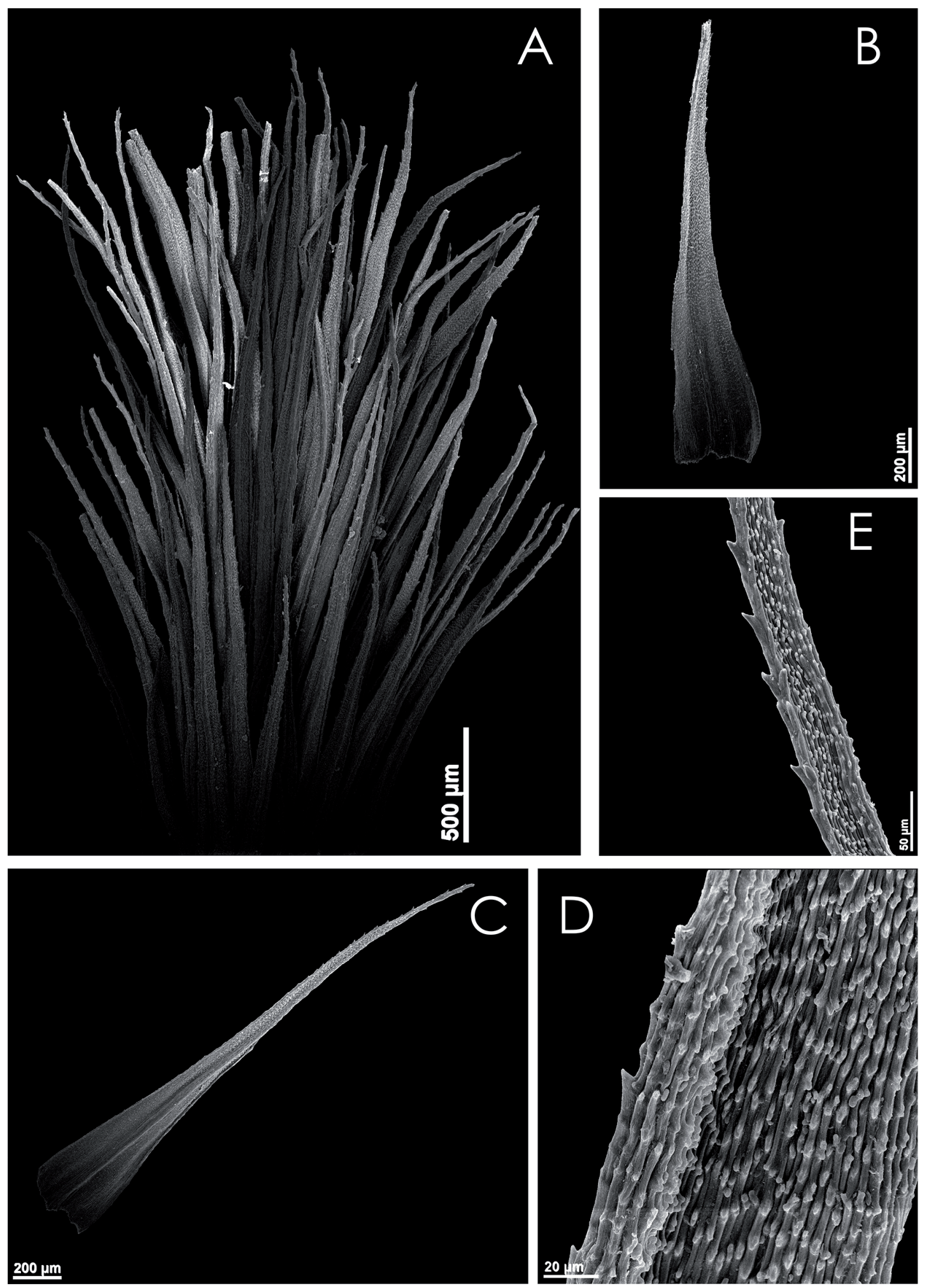

Figure 2. A. Detail of Bartramia laevisphaera on SEM. B-C. Leaves on SEM. D. Median leaf cells. E. Apical cells [from G. Suárez et al. 1366 (CTES, LIL)]. / Detalle de Bartramia laevisphaera en SEM. B-C. Hojas en SEM. D. Células de la lámina media. E. Células apicales [de G. Suárez et al. 1366 (CTES, LIL)]. 

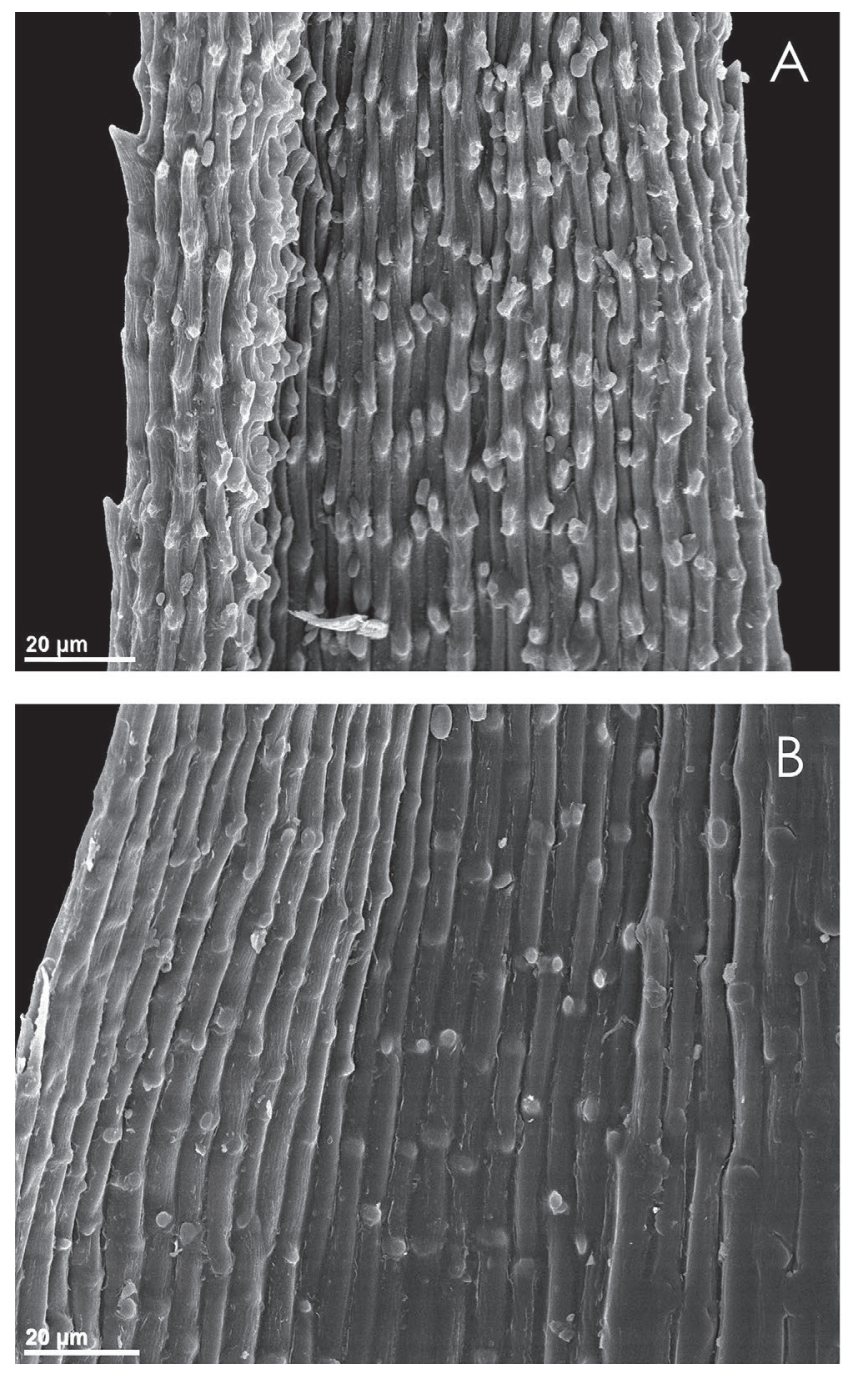

Figure 3. A. B. Adaxial surface of vegetative leaf on SEM [from G. Suárez et al. 1366 (CTES, LIL)]. / A. B. Superficie adaxial de la hoja en SEM [de G. Suárez et al. 1366 (CTES, LIL)].

\section{ACKNOWLEDGEMENTS}

Thanks are owed to the curators of the herbaria cited for making specimens available for examination. This research was sponsored by the Consejo Nacional de Investigaciones Científicas y Técnicas (CONICET), PICT (1838) and PIUNT (G524) from Argentina.

\section{REFERENCES}

Damayanti, L., Muñoz, J., Wicke, S., Symmank, L., Shaw, B., Frahm, J.-P., Quandt, D. 2012. Common but new:

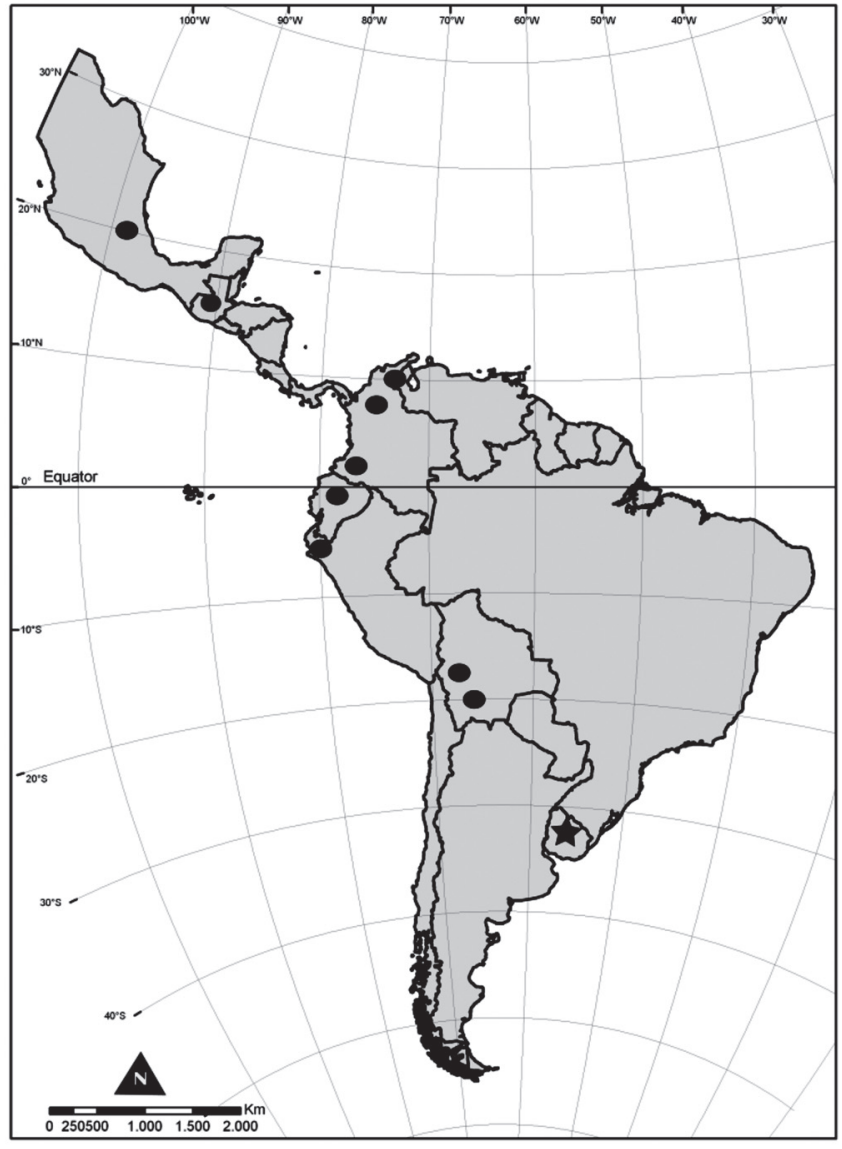

FIGURE 4. Distribution map of Bartramia laevisphaera in Latin America. Previous records are marked with dots, and the new record with a star. / Mapa de distribución de Bartramia laevisphaera en América Latina. Registros previos con círculos y nuevo el registro con una estrella.

Bartramia rosamrosiae, a "new" widespread species of apple mosses (Bartramiales, Bryophytina) from the Mediterranean and western North America. Phytotaxa 73: 37-59.

Ellis, L.T., Alegro, A., Bednarek-Ochyra, H., Ochyra, R., Bergamini, A., Cogoni, A., Erzberger, P., Górski, P., Gremmen, N., Hespanhol, H., Vieira, C., Kurbatova, L.E., Lebouvier, M., Martinčič, A., Asthana, A.K., Gupta, R., Nath, V., Natcheva, R., Ganeva, A., ÖZdemir, T., Batan, N., Plášek, V., Porley, R.D., Randić, M., Sawicki, J., Schroder, W., Sérgio, C., Smith, V.R., Sollman, P., Stefănut, S., Stevenson, C.R., SuÁrez, G.M., Surina, B., Uyar, G., Modrič Surina, Z. 2012a. New national and regional bryophyte records, 31. Journal of Bryology 34(2): 123-134. 
Ellis, L.T., Asthana, A.K., Sahu, V., Srivastava, A., BednarekOchyra, H., Ochyra, R., Chlachula, J., Colotti, M.T., Schiavone, M.M., Hradilek, Z., Jimenez, M.S., Klama, H., Lebouvier, M., Natcheva, R., Pócs, T., Porley, R.D., Sérgio, C., Sim-Sim, M., Smith, V.R., Söderström, L., Stefănut, S., SuÁrez, G.M., VÁnǍ, J. 2011. New national and regional bryophyte records, 28. Journal of Bryology 33(3): 237-47.

Ellis, L.T., Bednarek-Ochyra, H., Ochyra, R., Cykowska, B., Dulin, M.V., Ezer, T., Kara, R., Flores, J.R., SuÁrez, G.M., Garcia, C., Martins, A., Sérgio, C., Garilleti, R., Kirmaci, M., Agcagil, E., Kurbatova, L.E., Lebouvier, M., Papp, B., Szurdoki, E., Philippov, D.A., PlásěK, V., Pócs, T., Sabovljević, M., Sawicki, J., Sim-Sim, M., Szücs, P., Bidló, A., VÁnă, J., Vigalondo, B., Lara, F., Draper, I., Virchenko, V.M., Wolski, G.J. 2012b. New national and regional bryophyte records 33. Journal of Bryology 34(4): 281-291.
Flores, J.R., SuÁrez, G.M. 2014. Redescription of the genus Cryphidium (Cryphaeaceae, Bryophyta), with notes on its taxonomy. Boletín de la Sociedad Argentina de Botánica 49(2): 195-199.

Matteri, C.M. 1983. Sobre Bartramia stricta (Musci, Bartramiaceae) en la región Andino-patagónica. Boletín de la Sociedad Argentina de Botánica 22: 231-241.

Matteri, C.M. 2004. The mosses (Bryophyte) of Uruguay, their synonymy and distribution. Cryptogamie, Bryologie 25(2): 147-167.

SuÁrez, G.M., Schiavone, M.M. 2013. Contributions to the moss flora of Uruguay: The genus Pohlia (Bryophyta, Bryales), a new record. Darwiniana, nueva serie 1(1): 61-66.

SuÁrez, G.M., Schiavone, M.M., Colotti, M.T. 2014. The genus Holomitrium (Dicranaceae, Bryophyta) in Argentina and Uruguay. Boletín de la Sociedad Argentina de Botánica 49(2): 463-471.

Recibido: 30.09 .2016

Aceptado: 28.10 .2016 